\title{
Using Three-Dimensional Computational Modeling to Compare the Geometrical Fitness of Two Kinds of Proximal Femoral Intramedullary Nail for Chinese Femur
}

\author{
Sheng Zhang, ${ }^{1}$ Kairui Zhang, ${ }^{1}$ Yimin Wang, ${ }^{1}$ Wei Feng, ${ }^{2}$ Bowei Wang, ${ }^{1}$ and Bin Yu ${ }^{1}$ \\ ${ }^{1}$ Department of Orthopedics and Traumatology, Nanfang Hospital, Southern Medical University, Guangzhou 510515, China \\ ${ }^{2}$ Department of Orthopedics, Second Affiliated Hospital to Inner Mongolia Medical University, Hohhot 10020, China \\ Correspondence should be addressed to Bin Yu; 280219927@qq.com
}

Received 2 November 2012; Accepted 18 December 2012

Academic Editors: F. Leung, F. Liu, C. Morrey, and C.-W. Oh

Copyright (C) 2013 Sheng Zhang et al. This is an open access article distributed under the Creative Commons Attribution License, which permits unrestricted use, distribution, and reproduction in any medium, provided the original work is properly cited.

\begin{abstract}
Objective. The aim of this study was to use three-dimensional (3D) computational modeling to compare the geometric fitness of these two kinds of proximal femoral intramedullary nails in the Chinese femurs. Computed tomography (CT) scans of a total of 120 normal adult Chinese cadaveric femurs were collected for analysis. With the three-dimensional (3D) computational technology, the anatomical fitness between the nail and bone was quantified according to the impingement incidence, maximum thicknesses and lengths by which the nail was protruding into the cortex in the virtual bone model, respectively, at the proximal, middle, and distal portions of the implant in the femur. The results showed that PFNA-II may fit better for the Chinese proximal femurs than InterTan, and the distal portion of InterTan may perform better than that of PFNA-II; the anatomic fitness of both nails for Chinese patients may not be very satisfactory. As a result, both implants need further modifications to meet the needs of the Chinese population.
\end{abstract}

\section{Introduction}

Intramedullary fixation device has advantages from the biomechanical point of view and has become increasingly popular in treating unstable trochanteric fractures $[1,2]$, but serious implant-related complications have been reported, such as femoral shaft fracture [3], cutting out [4], lateral migration of the femoral neck screw $[4,5]$, and distal locking $[6,7]$. In 2003, the AO/ASIF group developed the Proximal Femoral Nail Antirotation (PFNA) to improve the rotational and angular stability with a helical blade which can avoid bone loss that occurs during the drilling and insertion of a standard sliding hip screw [8]. Biomechanical tests also demonstrated a significantly higher cut out resistance in the osteoporotic bone compared to commonly used screw systems $[9,10]$. However, lots of intra- and postoperative complications were reported since it was used in Asians, such as pain around the hip and the thigh, femoral shaft fracture, lateral blade migration, and lateral cortex splitting during operation $[9,11,12]$. In response, $\mathrm{AO} / \mathrm{ASIF}$ developed the PFNA-II, which was developed specifically for Asians. The flat lateral shape at the proximal portion of PFNA-II made it easier to be inserted intraoperatively, and its shortened proximal tip significantly reduced the postoperative hip pain $[13,14]$, but some Asian patients still complained about thigh pain after operation.

Similarly, a new device has been developed which uses 2 cephalocervical screws in an integrated mechanism allowing linear intraoperative compression and rotational stability of the head/neck fragment (InterTan) [15]. Biomechanical study shows the InterTan possesses some biomechanical benefits in internal fixation of unstable femoral neck fractures compared with DHS and CS [16]. Meanwhile, clinical outcomes support that the InterTan device appears to be a reliable implant for the treatment of intertrochanteric femoral fracture [15]. But few studies have reported the use of these two implants in Asians. The geometrical fitness between these two implants and Chinese femurs is still uncertain.

The purpose of this study was to compare the geometrical fitness of PFNA-II and InterTan for Chinese femurs and to discuss possible improvements needed for these two implants used in the Chinese population. We used three-dimensional (3D) computational modeling, because it has been well demonstrated that a $3 \mathrm{D}$ modeling is more precise than $2 \mathrm{D}$ 


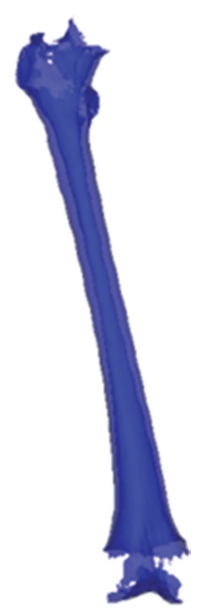

(a)

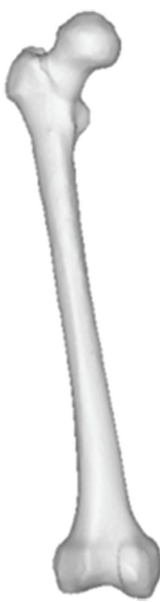

(b)

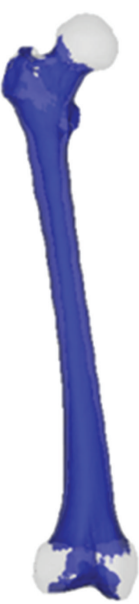

(c)

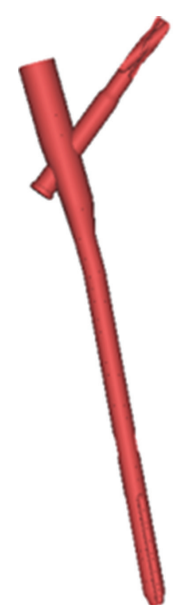

(d)

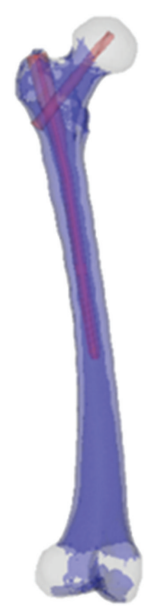

(e)

FIgure 1: The femur and implant models were reconstructed with software and assembled according to the paper guidelines. (a) The femoral cortex model; (b) the intact femur model. (c) The femoral cortex differentiated from the intact femur model. (d) The PFNA-II model; (e) the implant assembled with the femoral model.

methods by Budge et al. as it is less influenced by body position [17].

\section{Methods}

A total of 120 cadaveric specimens of full length femur were selected and CT scanned at Nanfang Hospital, Southern Medical University, Guangzhou, China. The scanning region was set from the top of the great trochanter at $0.75 \mathrm{~mm}$ intervals to the distal femoral joint surface.

The characteristics of the GE 16 row spiral CT scanner were as follows: $120 \mathrm{KV}, 300 \mathrm{MA}$, thickness of $0.75 \mathrm{~mm}$, pitch of $0.75 \mathrm{~mm}$, scan time of $200 \mathrm{~ms}$ each (10-12 s total scan time), and window diameter of $360 \mathrm{~mm}$. All CT data were saved in "dicom" format and imported into reverse engineering software Mimics 10.01 (Materialise, Belgium) for $3 \mathrm{D}$ reconstructions. 3D femoral models were established via masks creating, region growing, calculation, and remeshing of 3D objects.

According to the PFNA-II morphological parameters provided by Synthes and the InterTan morphological parameters provided by Smith \& Nephew, 3D models of the two implants were reconstructed with Pro E 4.0 (PTC, MA, USA) software. Then the models of both implants were assembled into the femoral models to compare the geometrical fitness between the implants and the femur (Figure 1).

2.1. Position of Implants. The specific location of the whole implant was adjusted until it was in full compliance with the manufacturer's operational guidelines. The antirotation screw was placed in the middle of the femoral head, and the depth of insertion of the intramedullary nail was determined according to the position of the antirotation nail.

2.2. Insertion Point. The entry point for the nail was defined according to the manufacturer's guidelines. In the anteroposterior position (AP) view, the entry point was at the vertex of the great trochanter. In the lateral view, the entry point was at the anterior $1 / 3$ of the vertex of the great trochanter.

In an ideal case, an anatomically shaped nail fits entirely the inside medullary cavity of the bone, indicating that the bone-nail construct stability is optimal, and the axial anatomical alignment of the bone is preserved. Therefore, in this study, the anatomical fitness between nail and bone was assessed by the extent the nail model was impinging or deviating from the medullary cavity of a particular intact (or nonfractured) femoral model. Once an impingement occurred, its incidence, thickness, and length were recorded precisely.

The following geometrical fitness parameters were precisely measured (Figures 2 and 3):

(A) The greatest thickness an implant impinged on the inner cortex of proximal femoral medullary cavity.

(B) The average length of an impingement area in proximal femur.

(C) The distance from the center of an impingement area (proximal) to the top of the great trochanter.

(D) The length of an implant protruding beyond the top of the great trochanter.

(E) The length and maximum thickness of a nail impinging the femoral shaft.

(F) The distance from the center of an impingement area to the top of the great trochanter (middle).

(G) The maximum distance between the inner cortex and an implant in the middle of femoral shaft.

(H) The length of an impingement area at the distal part of an implant.

2.3. Statistical Analysis. SPSS 16.0 (IBM, USA) was used for statistical analysis. Measurement data are shown in the form of mean $(\bar{X}) \pm \mathrm{SD}$. The repeated measures test was used to 


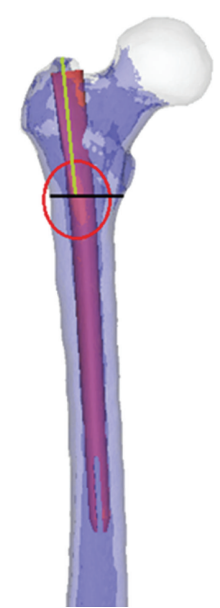

(a)

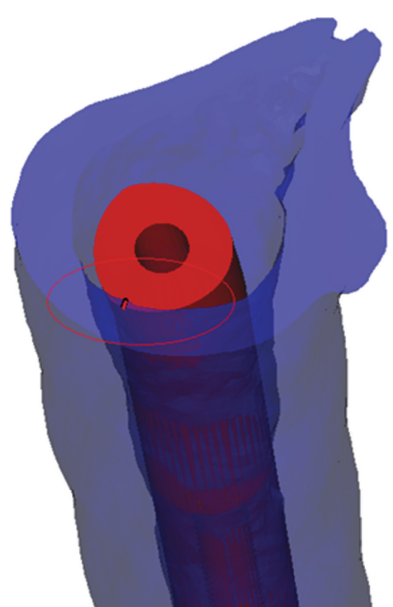

(b)

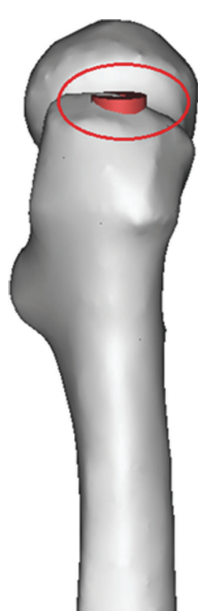

(c)

Figure 2: Proximal fitness of the implant with the femoral model. (a) The InterTan assembled with femoral model; the region within the red circle was the region of interest, the model was cut along the black line, and the distance between the top of the great trochanter and the center of the impingement region was depicted in green line. (b) The red line indicated the maximum thickness of the impingement area. (c) Within region the red circle was the proximal end of the implant protruding from the top of the great trochanter.

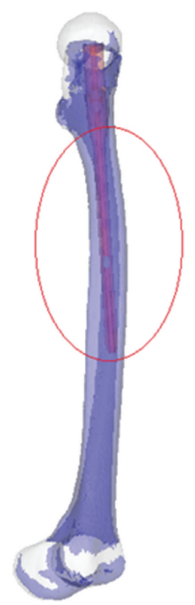

(a)

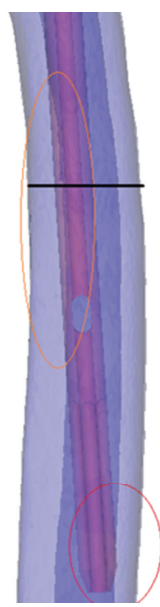

(b)

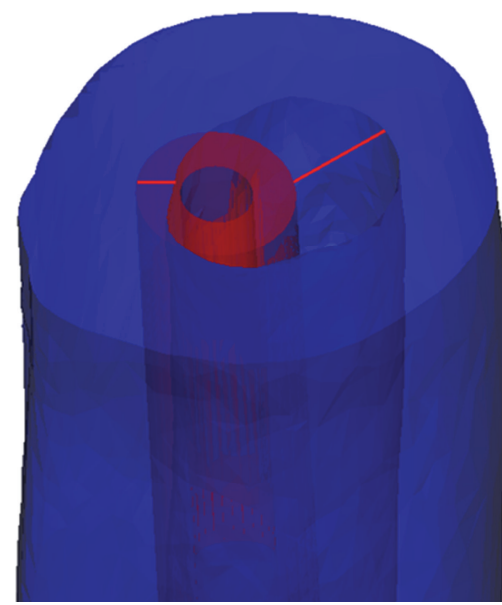

(c)

Figure 3: The model was assembled by PFNA-II and femur. (a) The region within the red circle was the region of interest. (b) The impingement area can be easily distinguished within the red circle, and the model was cut along the black line; (c) after the cut of the model, the maximum thickness of the impingement area and the distance from the inner cortex can be measured according to the direction of the red line.

assess the statistical significance of the measurement data. For all tests, $P<0.05$ was considered significant. Categorical variables were analyzed by the chi-square test or Fisher exact test where appropriate.

\section{Results}

3.1. Proximal Fitness. At the proximal part of the nail, the anatomical fitness of these two implants was assessed by criteria (A), (B), (C), and (D). The impingement occurred in $24.2 \%(n=29)$ of the InterTan models with an average thickness of $2.53 \mathrm{~mm}$ and an average length of $8.61 \mathrm{~mm}$, including 26 cases on the anterior side of the proximal femur and 3 on the lateral. In contrast, the impingement occurred only in 5.8\% $(n=7)$ of the PFNA-II models with an average thickness of $1.27 \mathrm{~mm}$ and an average length of $10.62 \mathrm{~mm}$, all on the lateral side of the proximal femur. There was a significant difference regarding the impingement incidence between the two models $(P=0.003)$. The average distance from the top of the great trochanter to the center of the impingement area was $57.31 \mathrm{~mm}$ in InterTan nails and $62.82 \mathrm{~mm}$ in PFNA-II (Table 1).

The incidence of the implants protruding beyond the top of the great trochanter was 5.8\% $(n=7)$ in InterTan nails and 7.5\% $(n=9)$ in PFNA-II, the average protruding lenths being $1.05 \mathrm{~mm}$ and $1.48 \mathrm{~mm}$, respectively.

3.2. Middle Fitness. The fitness at the middle portion of the implant for the femur was analyzed by the values (E), (F) and $(\mathrm{G})$. The impingement occurred in $53.3 \%(n=64)$ of 
TABLE 1: The average fitness value of impingement on the proximal portion of the implant ( $\mathrm{mm})$.

\begin{tabular}{|c|c|c|c|}
\hline & InterTan $(n=29)$ & PFNA-II $(n=7)$ & $P$ value \\
\hline Thickness & $2.53 \pm 0.58$ & $1.27 \pm 0.15$ & 0.017 \\
\hline Length & $8.61 \pm 1.84$ & $10.62 \pm 2.26$ & 0.183 \\
\hline Distance from the top of the great trochanter & $57.31 \pm 5.42$ & $62.82 \pm 5.87$ & 0.213 \\
\hline
\end{tabular}

TABLE 2: The average fitness value on the middle and distal portion of the implant $(\mathrm{mm})$.

\begin{tabular}{lcc}
\hline & $\begin{array}{c}\text { InterTan } \\
(n=64)\end{array}$ & $\begin{array}{c}\text { PFNA-II } \\
(n=52)\end{array}$ \\
\hline $\begin{array}{l}\text { Impingement thickness on the middle shaft of } \\
\text { the femur }\end{array}$ & $2.11 \pm 0.36$ & $1.62 \pm 0.38$ \\
$\begin{array}{l}\text { Impingement length on the middle shaft of the } \\
\text { femur }\end{array}$ & $60.62 \pm 8.37$ & $58.48 \pm 7.38$ \\
$\begin{array}{l}\text { Distance from the top of the great trochanter to } \\
\text { the central of the impingement area }\end{array}$ & $154.43 \pm 7.72$ & $148.74 \pm 8.87$ \\
$\begin{array}{l}\text { Distance between the nail and the inner cortex } \\
\text { in the lateral view }\end{array}$ & $\begin{array}{l}4.17 \pm 0.35 \\
(n=120)\end{array}$ & $\begin{array}{c}6.95 \pm 0.68 \\
(n=120)\end{array}$ \\
\hline
\end{tabular}

the InterTan models and $43.4 \%(n=52)$ of the PFNA-II models, and the incidences were comparable $(P=0.087)$. The parameters related to the impingement area were recorded in Table 2.

The maximum space between the implant and the inner cortex was larger in PFNA-II models than that in InterTan models. The average maximum space was $6.95 \mathrm{~mm}$ (SD: $0.68 \mathrm{~mm})$ in the PFNA-II models, much larger than in the InterTan models $(4.17 \mathrm{~mm} \pm 0.35 \mathrm{~mm})(P=0.035)$. In the lateral view, for all the models the maximum distance was located at the anterior edge of the nail (Figure 3).

3.3. Distal Fitness. At the distal part of the implant, impingement occurred in $31.7 \%(n=38)$ of the InterTan models, significantly lower than $52.5 \%$ in the PFNA-II models $(n=$ 63) $(P=0.007)$. The average length of impingement area was significantly shorter in the InterTan models $(8.5 \pm 2.13 \mathrm{~mm})$ than in the PFNA-II models $(17.6 \pm 4.37 \mathrm{~mm})(P=0.001)$.

\section{Discussion}

Intraoperative and postoperative complications associated with intramedullary nails in the treatment of intertrochanteric fracture can result from imperfect fitness of an internal implant with specific morphology of the human bone. Design of anatomically fitting nails requires morphologic data of the bone that are representative of a specific population. It is well known that fixation devices based on the geometrical proportions of Caucasians do not adequately fit Asian patients, leading to such device-related complications as reported for the Gamma nail [18] and ETN [19].

It has been well demonstrated that the PFNA with a bending angle of $6^{\circ}$ and a proximal diameter of $17 \mathrm{~mm}$ would cause a fracture of the proximal femur during inserting the nail. Otherwise, a larger femoral cannel should be prepared to benefit the nail insert more smoothly $[12,20]$. The PFNAII for Asians was designed to have a bending angle of $5^{\circ}$ and a proximal diameter of $16.5 \mathrm{~mm}$. Additionally, the proximal lateral surface was made flat to facilitate insertion and to lower the pressure on the lateral cortex [13]. The present study has demonstrated that the modified nail has a considerably better anatomic fitness for Chinese proximal femur. On the contrary, in this study the InterTan performed worse in the geometrical fitness with Chinese proximal femur, with an impingement incidence of $24.2 \%$. Most impingements occurred on the anterior side of the proximal femur, indicating the anteroposterior diameter of the InterTan may not match well Chinese proximal femurs. Additionally, it is not easy to insert an InterTan nail with a trapezoidal proximal end into a poorly reduced marrow cavity. Consequently, repeated reduction and manipulation may increase both operative and fluoroscopy time and blood loss as well.

Hip and thigh pain $[10,21]$ seemed to be very common complications in previous reports and could occur in $90.1 \%$ of the cases during the follow-up period. One explanation comes to the chronic muscle injury caused by the overlong proximal end of the nail protruding beyond the great trochanter. In this study, both implants matched well the proximal tip of the great trochanter. Clinical results also supported that few patients experienced tenderness following either PFNA-II or InterTan implantation [10, 15].

It is known that Chinese women have a shorter femoral neck, smaller femoral neck angles, and increased anterior bowing of the shaft than white American women $[18,19]$. It is also acknowledged that the overall stability of the boneimplant construct is important for significant improvements in decreasing postoperative pain and increasing postoperative walking ability. In this study, however, both nails impinged frequently upon the posterior side of the femur at the middle portion of the implant but deviated a lot from the anterior inner cortex from the lateral view. Moreover, both nails did not contact very well the inner cortex at the middle shaft of the femur, due to the Asian characteristic anterior bowing of the femoral shaft. As a result, stress can be concentrated on this region, especially around the locking screw, making the femoral shaft more vulnerable to fracture 
or leading to long-term thigh pain that cannot be explained. It is suggested that the morphology of the Chinese anterior bowing of the femoral shaft should be taken into consideration when these two nails are used in Asians, especially for those shorter elderly female patients. Furthermore, moderate modification is needed for both implants.

Furthermore, in the present study both implants were biased to the anterior inner cortex at the distal portion from the lateral view. The length of the impingement area was longer in PFNA-II models than in InterTan models. In a similar way, stress may be usually concentrated on this area. It is advised that a shorter PFNA-II should be chosen in treatment of short elderly female patients.

In this study, the impinged areas were mostly located in three regions of the femur. The first region was about $60 \mathrm{~mm}$ below the top of the great trochanter and on the anterolateral side of the proximal femur. The second one was located on the posterior side of the femoral shaft, about $155 \mathrm{~mm}$ below the top of the great trochanter. The third region was around the distal tip of the nail. In order to design a proximal femoral intramedullary nail fit for Chinese population, the three above regions should also be taken into consideration.

The impressive strength of the present study lies in the simplicity, efficiency, and low cost of the methodology we used to compare the fitness of two implants for a given population, compared with clinical trials.

In conclusion, although PFNA-II may fit better for the Chinese proximal femurs than InterTan and the distal portion of InterTan may perform better than that of PFNA-II, the anatomic fitness of both nails for Chinese patients may not be very satisfactory. Further clinical results are needed to test the findings of the present 3D computational modeling. In addition, multicenter CT data are also needed to build a database of Chinese bones for 3D modeling which will serve as the basis necessary for the research and development of orthopedic devices for the Chinese population.

\section{Conflict of Interests}

The authors declare that they have no conflict of interests.

\section{Authors Contribution}

S. Zhang, K. Zhang, and Y. Wang contributed equally to this work.

\section{Acknowledgments}

The authors thank Professor Liang Ping for his aid in revising the present paper. This study was supported by the National Natural Science Fund of China (81071233) and a combination of the project of the Ministry of Education and Guangdong Province (2009B090300279).

\section{References}

[1] E. N. Kubiak, M. Bong, S. S. Park, F. Kummer, K. Egol, and K. J. Koval, "Intramedullary fixation of unstable intertrochanteric hip fractures: one or two lag screws," Journal of Orthopaedic Trauma, vol. 18, no. 1, pp. 12-17, 2004.

[2] F. Loubignac and J. F. Chabas, "A newly designed locked intramedullary nail for trochanteric hip fractures fixation: results of the first 100 TrochantericU implantations," Orthopaedics and Traumatology: Surgery and Research, vol. 95, no. 2, pp. 139-144, 2009.

[3] J. Albareda, A. Laderiga, D. Palanca, L. Paniagua, and F. Seral, "Complications and technical problems with the gamma nail," International Orthopaedics, vol. 20, no. 1, pp. 47-50, 1996.

[4] I. B. Schipper, E. W. Steyerberg, R. M. Castelein et al., "Treatment of unstable trochanteric fractures. Randomised comparison of the gamma nail and the proximal femoral nail," Journal of Bone and Joint Surgery. British, vol. 86, no. 1, pp. 8694, 2004.

[5] M. Tyllianakis, A. Panagopoulos, A. Papadopoulos, S. Papasimos, and K. Mousafiris, "Treatment of extracapsular hip fractures with the proximal femoral nail (PFN): long term results in 45 patients," Acta Orthopaedica Belgica, vol. 70, no. 5, pp. 444454, 2004.

[6] P. Maniscalco, F. Rivera, J. D’Ascola, and E. O. Del Vecchio, "Failure of intertrochanteric nailing due to distal nail jamming," Journal of Orthopaedics and Traumatology. In press.

[7] J. Windolf, D. A. Hollander, M. Hakimi, and W. Linhart, "Pitfalls and complications in the use of the proximal femoral nail," Langenbeck's Archives of Surgery, vol. 390, no. 1, pp. 59-65, 2005.

[8] P. Mereddy, S. Kamath, M. Ramakrishnan, H. Malik, and N. Donnachie, "The AO/ASIF proximal femoral nail antirotation (PFNA): a new design for the treatment of unstable proximal femoral fractures," Injury, vol. 40, no. 4, pp. 428-432, 2009.

[9] A. Brunner, J. A. Jöckel, and R. Babst, "The PFNA proximal femur nail in treatment of unstable proximal femur fractures3 cases of postoperative perforation of the helical blade into the hip joint," Journal of Orthopaedic Trauma, vol. 22, no. 10, pp. 731-736, 2008.

[10] X. Yaozeng, G. Dechun, Y. Huilin, Z. Guangming, and W. Xianbin, "Comparative study of trochanteric fracture treated with the proximal femoral nail anti-rotation and the third generation of gamma nail," Injury, vol. 41, no. 12, pp. 1234-1238, 2010.

[11] J. S. Pu, L. Liu, G. L. Wang, Y. Fang, and T. F. Yang, "Results of the proximal femoral nail anti-rotation (PFNA) in elderly Chinese patients," International Orthopaedics, vol. 33, no. 5, pp. 1441-1444, 2009.

[12] J. H. Hwang, J. K. Oh, S. H. Han, W. Y. Shon, and C. W. $\mathrm{Oh}$, "Mismatch between PFNa and medullary canal causing difficulty in nailing of the pertrochanteric fractures," Archives of Orthopaedic and Trauma Surgery, vol. 128, no. 12, pp. 14431446, 2008.

[13] C. Lv, Y. Fang, L. Liu et al., "The new proximal femoral nail antirotation-Asia: early results," Orthopedics, vol. 34, no. 5, article 351, pp. e18-e23, 2011.

[14] V. Tyagi, J. H. Yang, and K. J. Oh, "A computed tomographybased analysis of proximal femoral geometry for lateral impingement with two types of proximal femoral nail anterotation in subtrochanteric fractures," Injury, vol. 41, no. 8, pp. 857861,2010

[15] A. H. Ruecker, M. Rupprecht, M. Gruber et al., "The treatment of intertrochanteric fractures: results using an intramedullary nail with integrated cephalocervical screws and linear compression," Journal of Orthopaedic Trauma, vol. 23, no. 1, pp. 22-30, 2009. 
[16] M. Rupprecht, L. Grossterlinden, A. H. Ruecker et al., "A comparative biomechanical analysis of fixation devices for unstable femoral neck fractures: the Intertan versus cannulated screws or a dynamic hip screw," The Journal of Trauma, vol. 71, no. 3, pp. 625-634, 2011.

[17] M. D. Budge, G. S. Lewis, E. Schaefer, S. Coquia, D. J. Flemming, and A. D. Armstrong, "Comparison of standard twodimensional and three-dimensional corrected glenoid version measurements," Journal of Shoulder and Elbow Surgery, vol. 20, no. 4, pp. 577-583, 2011.

[18] W. M. Tang, K. Y. Chiu, M. F. Y. Kwan, T. P. Ng, and W. P. Yau, "Sagittal bowing of the distal femur in Chinese patients who require total knee arthroplasty," Journal of Orthopaedic Research, vol. 23, no. 1, pp. 41-45, 2005.

[19] K. S. Leung, P. Procter, B. Robioneck, and K. Behrens, "Geometric mismatch of the gamma nail to the Chinese femur," Clinical Orthopaedics and Related Research, no. 323, pp. 42-48, 1996.

[20] R. K. J. Simmermacher, J. Ljungqvist, H. Bail et al., "The new proximal femoral nail antirotation (PFNA) in daily practice: results of a multicentre clinical study," Injury, vol. 39, no. 8, pp. 932-939, 2008.

[21] B. Garg, K. Marimuthu, V. Kumar, R. Malhotra, and P. P. Kotwal, "Outcome of short proximal femoral nail antirotation and dynamic hip screw for fixation of unstable trochanteric fractures. A randomised prospective comparative trial," Hip International, vol. 21, no. 5, pp. 531-536, 2011. 


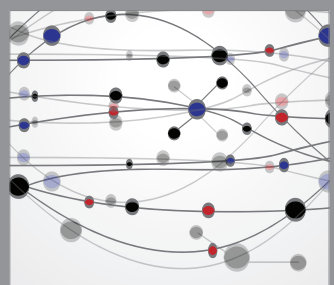

The Scientific World Journal
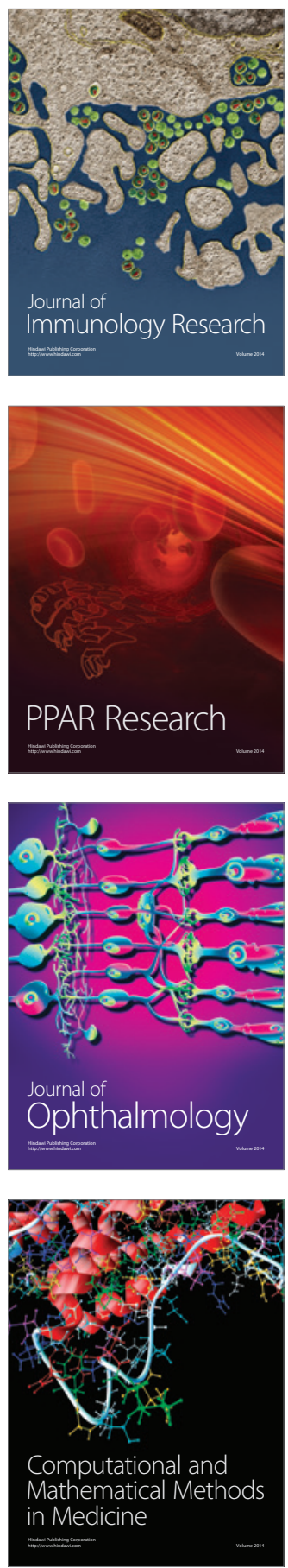

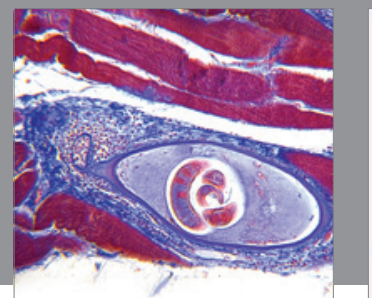

Gastroenterology

Research and Practice
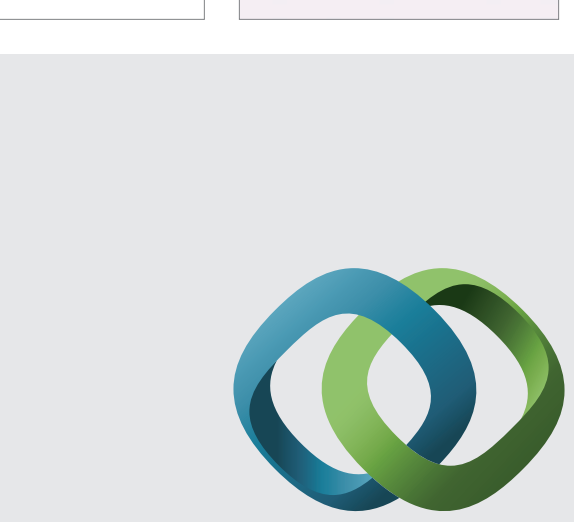

\section{Hindawi}

Submit your manuscripts at

http://www.hindawi.com
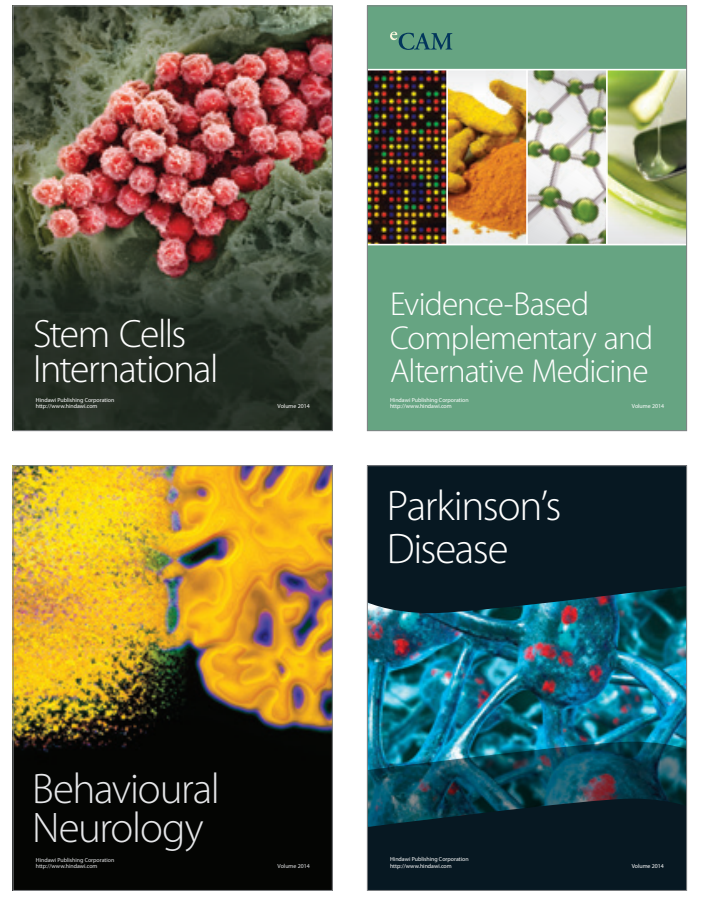
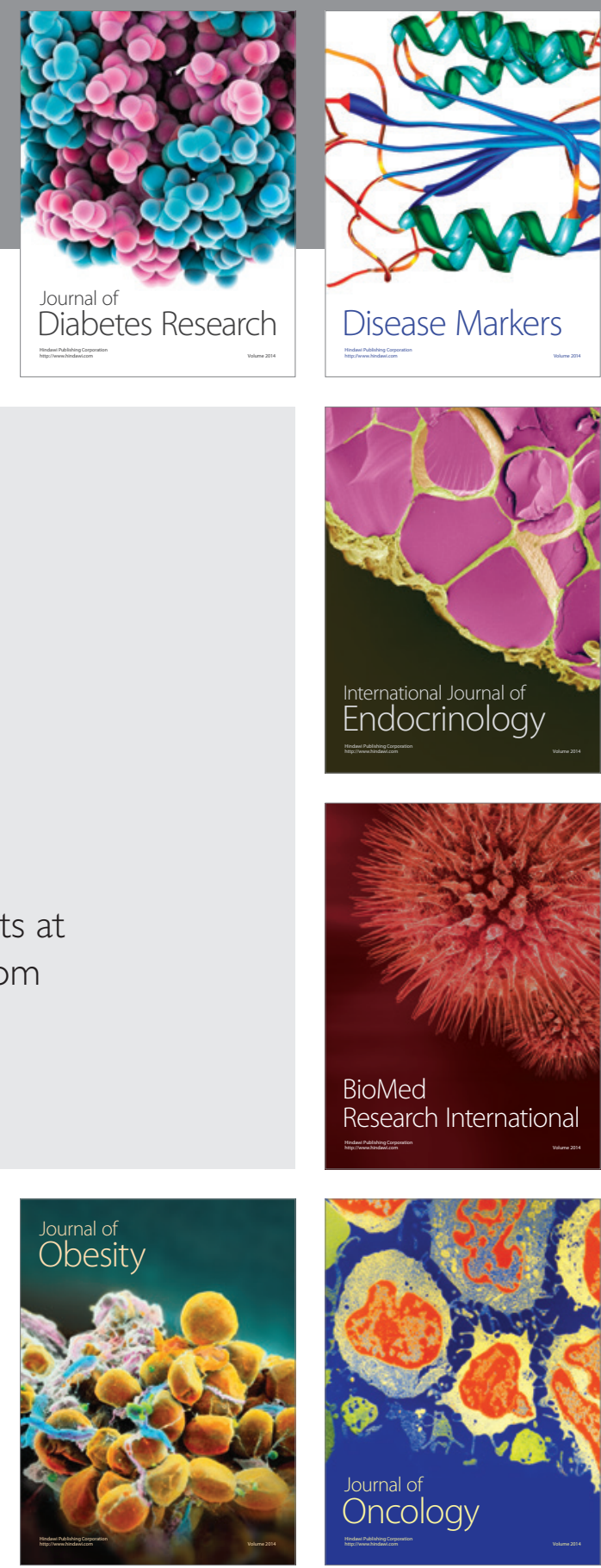

Disease Markers
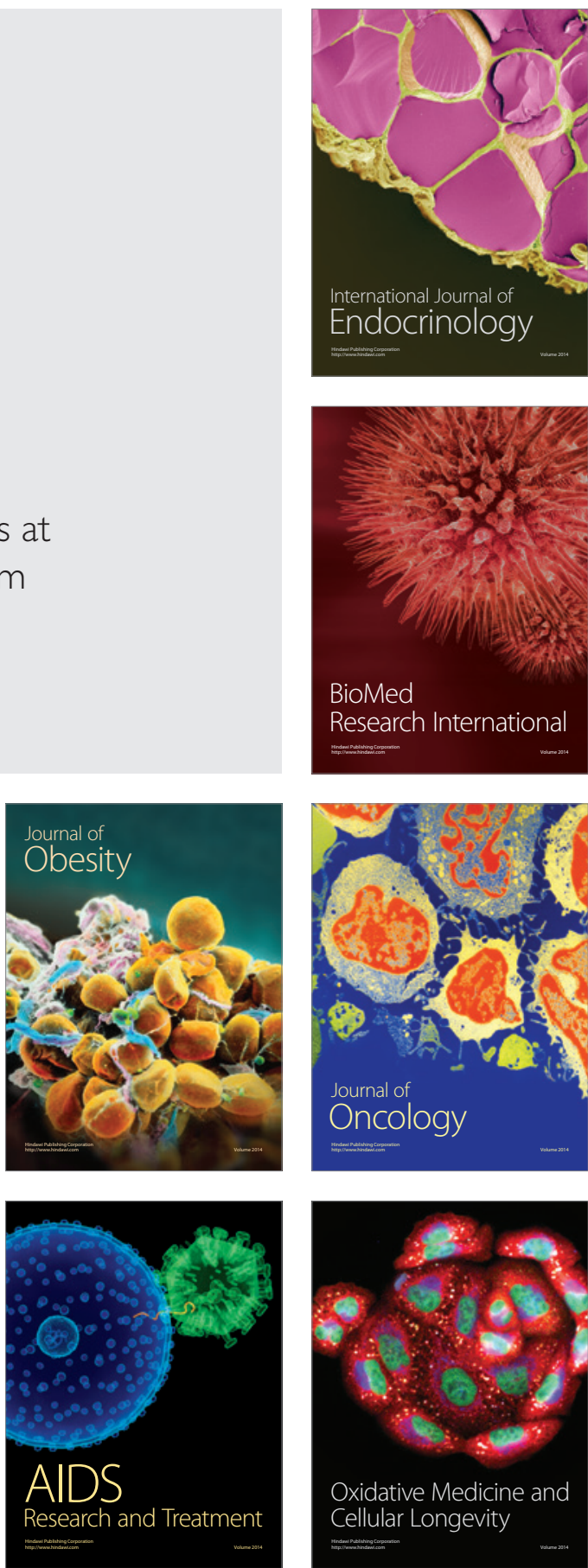